\title{
Improving the completion of Quality Improvement projects amongst psychiatry core trainees
}

\author{
Liz Ewins \\ Medical Education, Avon and Wiltshire Mental Health Partnership Trust
}

\begin{abstract}
Quality Improvement (QI) projects are seen increasingly as more valuable and effective in developing services than traditional audit. However, the development of this methodology has been slower in the mental health field and QI projects are new to most psychiatrists. This project describes a way of engaging trainees across Avon and Wiltshire Mental Health Partnership (AWP) Trust and the Severn School of Psychiatry in QI projects, using QI methodology itself.

Through the implementation and development of training sessions and simple, low cost and sustainable interventions over a 10 month period, two thirds of core trainees and over a half of the advanced psychiatry trainees in the School are now participating in 28 individual QI projects and QI project methodology is to become embedded in the core psychiatry training course. As an additional positive outcome, specialty doctors, consultants, foundation doctors, GP trainees, medical students, as well as the wider multidisciplinary team, have all become engaged in QI projects alongside trainees, working with service users and their families to identify problems to tackle and ideas to test.
\end{abstract}

\section{Problem}

Quality Improvement (QI) projects can lead to real changes in patient care in terms of improving safety, efficiency and patient experience. They also provide trainees with leadership and management experience and opportunities to present and publish work.

However, the development of this methodology has been slower in the mental health field and QI projects are new to most psychiatrists. Here we outline and summarise in simple steps how to undertake a QI project. We also describe our cross-Deanery project led in Avon and Wiltshire Trust and the Severn School of Psychiatry, which aims to train and support psychiatrists of the future to become actively engaged in QI projects.

\section{Background}

Foundation doctors and Core Medical Trainees are being trained to undertake QI projects and are doing so competently and effectively $[1,2]$. QI projects have been embedded in the Foundation Programme curriculum [3] with an expectation that trainees plan, implement, complete, and present a QI project as part of their training, using the results to improve patient care. QI projects have been widely adopted across medicine and are in many areas superseding traditional audit as a way to develop services. QI projects often lead to real changes in patient care, and provide leadership and management experience to trainees as well as opportunities to give presentations and complete publications.

QI projects aim to improve patient safety, treatment effectiveness and efficiency, and the patient experience. QI projects are real-time, dynamic processes involving focused, progressive, small scale changes through a simple structured framework, which enable visible and effective change over a short period of time. QI projects are becoming increasingly important because of the limited resources available in the NHS. They can support service change and to achieve this may engage all professional groups, including trainees. Increasingly, evidence of QI is becoming a training requirement and is taking the place of audit as a subject to discuss at trainee reviews and job interviews.

\section{Baseline measurement}

For this project both quantitative and qualitative data was considered. The first measure is the number of QI projects registered in the Deanery. The second measure is the number of trainees and other professionals involved in QI projects. Additionally, qualitative data will be gathered from trainees and others to further evaluate the impact of changes made.

At baseline, there was no formal way of monitoring the number of QI projects that core trainees are currently involved in. No QI projects were known to be occuring across the Deanery.

\section{Design}

When examining the underlying cause of the problem, it appeared that a lack of knowledge and understanding about QI and QI methodology was key. Education and training would therefore play a key role. A training session would be delivered by the author, a teaching fellow, AWP Medical Education and the QI Lead from AWP's Quality Academy. This would educate trainees in QI methodology and enable them to undertake their own QI project. Following this, monthly short training sessions could be delivered at the Deanery's Core Trainees' Course, which would support the 
trainees to develop and undertake their own QI projects.

The project aimed to be completed within 10 months, with training being provided initially in Autumn 2015. Once resources are set up, this training package could be continued by future teaching fellows. As training is provided by Trust and Deanery staff and based in Trust and Deanery buildings, costs will be kept to an absolute minimum.

\section{Strategy}

In the first PDSA cycle an online registration form was developed for trainees to register their QI projects. Trainees were informed of this by email, but as this was new to many and they had no knowledge of QI methodology, no forms were completed.

The second intervention consisted of delivering a teaching session about QI delivered by the author, AWP Medical Education and the QI Lead from AWP's Quality Academy. This was an optional session but was well attended by trainees (both core and advanced), as well as specialty doctors and consultants. As a result of this a small number of projects were registered using the online form.

The third PDSA cycle consisted of a repeat of the half day course, but this time as well as trainees being invited, consultants and tutors were specifically invited to attend. The course was developed to include specific, clinical examples, and more information on QI methodology. This resulted in a growing number of projects being registered.

The fourth intervention consisted of a follow-up teaching session being delivered at the trainees' Deanery core training course.

A further session was then delivered, but this time it took the form of a drop-in advice session to support trainees in their individual projects.

An email was then sent out from the Associate Director of Medical Education to all trainees reminding them to register and providing them with a repeat of information already sent out about QI projects.

Finally, a session was delivered by the author at the Trust Medical Advisory Group quarterly meeting. Ths introduced the programme of enabling trainees and briefly outlined QI methodology. This was to a large audience of consultants from across the Trust.

\section{Post-measurement}

Measurements were taken at baseline and then at monthly intervals over a period of 10 months. At baseline, no QI projects were registered and no trainees were engaged in QI projects.

Over the course of the programme, the number of projects registered and trainees engaged, as well as other professionals, including specialty doctors, consultants, foundation doctors, GP trainees, medical students, as well as the wider multidisciplinary team, increased. (Please see Fig. 1 attached)

By the end of 10 months, over two thirds of core trainees ( $n=21)$ and over half of advanced trainees $(n=16)$ in the Deanery were involved in a total of $28 \mathrm{QI}$ projects. A growing number of specialty doctors ( $n=54 ; 11 \%$ of the Trust's total) and consultants $(n=24$; $22 \%$ ) and colleagues from the wider multidisciplinary team, as well as foundation doctors and medical students are also becoming involved in projects.

Evaluation of the programme so far has shown globally positive feedback from consultants and high engagement and enjoyment from trainees. This is reported by trainees to be in part due to them being able to choose their own QI projects rather than being instructed what to do. Trainees report that training sessions and follow-up advice has been helpful and all feel that this should be provided to all staff. One trainee fed back that "the quality improvements often end up involving other team members so it would be good to get them on side" with another trainee highlighting that "training is needed for senior staff members who we may need to get on board." Projects frequently need senior input to facilitate implementation and sustainability of successful changes and several projects have needed advice around governance issues.

See supplementary file: ds5960.docx - "Fig.1. Graph to show the percentage of trainees and others undertaking QI projects"

\section{Lessons and limitations}

During the project I found that forming close links with the Trust essential. The Trust Quality Academy, responsible for audit and QI projects, provided invaluable assistance in setting up the scheme. The Academy has a QI project lead who provides advice and training for trainees. They have been particularly helpful when offering guidance regarding governance and what permissions might need to be sought.

Support from Trust medical management leads, including the medical director and director of medical education has also been key in facilitating not only this project, but trainees individual projects too.

The trainees projects have also highlighted that due to the nature of our work in mental health, QI projects sometimes need to be structured in a different way than they would be in an acute hospital setting. Careful consideration is needed to find the most appropriate method of change measurement, as frequently qualitative data may be available and quantitative data less so. We have found that the longer duration of inpatient stays sometimes results in QI projects running over a longer period of time than they would in an acute hospital.

As well as positively influencing patient care, trainees report that projects are providing them with invaluable opportunities for leadership and management experience, and many are beginning to present and publish their work. We are encouraging trainees to do so even if a project has not been fully successful, as much will 
have been and can be learned by the trainees and the Trust from all projects.

\section{Conclusion}

At the start of the project, no trainees were known to be engaged in QI projects and there was no way of capturing this data. The introduction of the registration form was necessary, however the training sessions and support to trainees and their individual projects was key in making this project a success. By supporting trainees to come up with their own ideas for projects, enjoyment and engagement was high. This project has had the additional benefit of being from a bottom-up approach, which has meant that as trainees have become involved, their seniors as well as other members of the multidisciplinary team have become involved too.

With foundation doctors and medics from across all specialties undertaking QI projects, it is important that psychiatrists become engaged in QI work too. This project has illustrated that simple, sustainable methods can be employed to enable trainees to undertake their own projects. As well as positively contributing to patient care, the projects have given trainees invaluable management and leadership knowledge and skills, as well as provided them with opportunities to present and publish their own work.

\section{References}

1. Bethune R, Soo E, Woodhead P, Van Hamel C, Watson C. Engaging all doctors in continuous quality improvement: a structured, supported programme for first-year doctors across a training deanery in England. BMJ Qual Saf 2013; 22(8):613-7.

2. Vaux E, Went $S$, Norris $M$, Ingham J. Learning to make a difference: introducing quality improvement methods to core medical trainees. Clin Med 2012; 12(6): 520-5.

3. United Kingdom Foundation Programme. The Foundation Programme Curriculum 2012. Available at http://www.foundationprogramme.nhs.uk/pages/home/curric ulum-and-assessment/curriculum2012

\section{Declaration of interests}

Nothing to declare.

\section{Acknowledgements}

I am grateful for the support of my project supervisors Dr Geoff van der Linden (Consultant QI Lead and Associate Director of Medical Education) and Dr Rob Macpherson (Head of School), as well as the Director of Medical Education, Dr Steve Arnott. I am also grateful to Janet Brandling and Emma Adams (AWP Quality Academy), Bridget Kelly (AWP Medical Education Project Manager) and Katherine Finucane (Consultant and QIP Lead, North Bristol Trust) for guidance and support in developing QI projects across the Deanery.

\section{Ethical approval}

Approval was sought and gained from the Director of Medical Education in AWP, the Head of School of Severn Deanery, and AWP's Medical Director. The project was discussed with the Deanery's Educational Fellowship team and as it was an improvement study no ethical approval was deemed necessary. 